\title{
Capillary Electrophoresis-Atmospheric Pressure Chemical Ionization-Mass Spectrometry Using an Orthogonal Interface: Set-Up and System Parameters
}

\author{
Paul Hommerson, ${ }^{a}$ Amjad M. Khan, ${ }^{\text {b Gerhardus J. de Jong, }}$, \\ and Govert W. Somsen ${ }^{\mathrm{a}}$ \\ ${ }^{a}$ Department of Biomedical Analysis, Utrecht University, Utrecht, The Netherlands \\ b Analytical Chemistry, Process R and D, AstraZeneca, Macclesfield, Cheshire, United Kingdom
}

\begin{abstract}
The feasibility of atmospheric pressure chemical ionization (APCI) as an alternative ionization technique for capillary electrophoresis-mass spectrometry (CE-MS) was investigated using a grounded sheath-flow CE-MS sprayer and an orthogonal APCI source. Infusion experiments indicated that highest analyte signals were achieved when the sprayer tip was in close vicinity of the vaporizer entrance. The APCI-MS set-up enabled detection of basic, neutral, and acidic compounds, whereas apolar and ionic compounds could not be detected. In the positive ion mode, analytes could be detected in the entire transfer voltage range $(0-5 \mathrm{kV})$, whereas highest signal intensities were observed when the corona discharge current was between 1000 and $2000 \mathrm{nA}$. In the negative ion mode, the transfer voltage typically was $500 \mathrm{~V}$ and the optimum corona discharge current was $6000 \mathrm{nA}$. Analyte signals were raised with increasing nebulizing gas pressure, but the pressure was limited to 25 psi to avoid siphoning and current drops. Signal intensities appeared to be optimal and constant over a wide range of sheath liquid flow rate $(5-25 \mu \mathrm{L} / \mathrm{min})$ and vaporizer temperature $\left(200-350^{\circ} \mathrm{C}\right)$. APCI-MS signals were unaffected by the composition of the background electrolyte (BGE), even when it contained sodium phosphate and sodium dodecyl sulfate (SDS). Consequently, BGE composition, sheath-liquid flow rate, and vaporizer temperature can be optimized with respect to the $\mathrm{CE}$ separation without affecting the APCI-MS response. The analysis of a mixture of basic compounds and a steroid using volatile and nonvolatile BGEs further demonstrates the feasibility of CE-APCIMS. Detection limits $(\mathrm{S} / \mathrm{N}=3)$ were 1.6-10 $\mu \mathrm{M}$ injected concentrations. (J Am Soc Mass Spectrom 2009, 20, 1311-1318) @ 2009 Published by Elsevier Inc. on behalf of American Society for Mass Spectrometry
\end{abstract}

\begin{abstract}
S ince its introduction in 1987 [1], capillary electrophoresis-mass spectrometry (CE-MS) has evolved into a powerful combination, which is now routinely applied for a number of analytical tasks. The coupling of CE and MS has predominantly been achieved using electrospray ionization (ESI)-based interfaces. This is because CE is commonly applied to compounds that are charged in solution and, therefore, inherently suited to ESI-MS. Still, ESI-MS entails a number of limitations. First, the ESI process is very susceptible for nonvolatile background electrolytes (BGEs) and constituents, and this restricts the choice of BGEs amenable to CE-MS. Second, effective ESI is limited to relatively polar compounds, and may therefore not be a suitable option when neutral and/or less polar compounds are to be analyzed. In an attempt to overcome these limitations, only few studies explored
\end{abstract}

Address reprint requests to P. Hommerson, Department of Biomedical Analysis, Utrecht University, P.O. Box 80082, 3508 TB Utrecht, The Netherlands. E-mail: P.Hommerson@uu.nl the potential of alternative ionization techniques for CE-MS, such as atmospheric pressure chemical ionization (APCI) [2-5] and, more recently, atmospheric pressure photoionization (APPI) [6-9]. Owing to its capability to form both even- and odd-electron ions, the latter technique was found suitable for ionization of both polar and apolar compounds. Furthermore, APPI appeared to be unaffected by the BGE composition, and even enabled the coupling of micellar electrokinetic chromatography (MEKC) and microemulsion electrokinetic chromatography (MEEKC) with MS [10, 11].

APCI is another ionization technique in which analyte ionization occurs through gas-phase ion-molecule reactions. In LC-MS, APCI is routinely used and regarded a strong alternative for ESI. The use of APCI in CE-MS, however, has been limited to a few explorative studies only. The possibility of CE-APCI-MS was indicated by Takada et al. [2], who employed a laboratorymade sheath-flow sprayer in combination with a commercially available APCI source. The signal intensity of the model compound caffeine was hardly affected 
when using a sodium phosphate BGE. However, the caffeine concentration $(1 \mathrm{mM})$ was not representative for most analytical tasks. Muijselaar et al. [3] evaluated APCI as a part of a study on partial-filling MEKC-MS. For caffeine and ethenzamide concentrations of 0.5 $\mathrm{mg} / \mathrm{mL}$ detectable signals were obtained. In a followup study by Isoo et al. [4], initial sample concentrations (100-1000 $\mu \mathrm{g} / \mathrm{mL}$ ) were also high, but a 100- to 600-fold enhancement in sensitivity was achieved using on-line sample concentration. In these preliminary studies, the nebulization of the $\mathrm{CE}$ effluent and sheath liquid relied on electrospray only, and probably efficient analyte introduction and/or transfer through the vaporizer was not achieved. Tanaka et al. [5] improved the CEAPCI-MS set-up by employing nebulizing gas to enhance the ionization process. Modification of the APCI source was required to achieve a sufficient penetration of the CE-MS sprayer into the vaporizer, and thus obtain an appreciable analyte signal. The feasibility of the set-up was briefly indicated by the analysis of a mixture of four basic test drugs $(25-50 \mu \mathrm{g} / \mathrm{mL})$.

In the present study, CE-APCI-MS is achieved without significant modifications of the equipment using an orthogonal ion source in combination with a commercial sheath-flow interface. In this set-up, the spray formation is independent of the transfer voltage and occurs orthogonally to the mass spectrometer inlet. As a result, the contribution of (undesirable) ESI processes to overall ionization is significantly reduced, which may lead to more selective ion formation and a lower background noise. This CE-APCI-MS set-up has not been studied before and, therefore, an exploration of the main operating parameters was needed. For this purpose, a set of model drugs, i.e., basic, acidic, neutral, apolar, and ionic (Figure 1), was used, thereby also evaluating the influence of analyte character. The effect of volatile and nonvolatile BGEs on the APCI-MS response was also investigated. Finally, the overall CEAPCI-MS performance was evaluated by the analysis of a mixture of test drugs.

\section{Experimental}

\section{Chemicals and Materials}

Sodium dihydrogen phosphate, disodium hydrogen phosphate, sodium dodecyl sulfate (SDS), sodium hydroxide, ammonium acetate, and ammonia solution (25\%) were supplied by Merck (Darmstadt, Germany). Formic acid, terbutaline, atropine, nicotine, caffeic acid, and diphenyl sulfide were purchased from SigmaAldrich (Zwijndrecht, The Netherlands). Methanol was from Biosolve (Valkenswaard, The Netherlands). Mebeverine was obtained from Solvay Pharmaceuticals (Weesp, The Netherlands). Methyl atropine, hydrocortisone, and propyl p-hydroxybenzoate (POB) were from Fagron (Nieuwerkerk a/d Ijssel, The Netherlands). Ultrapure water was obtained from a Synergy UV water system from Millipore (Molsheim, France). Fused-silica capillaries were from BGB Analytik (Boekten, Switzerland).

For the infusion experiments, a solution of diphenyl sulphide was prepared in methanol, whereas solutions of the other test compounds were prepared in water, in $50 \mathrm{mM}$ ammonium acetate ( $\mathrm{pH}$ 6.8), in $10 \mathrm{mM}$ sodium phosphate ( $\mathrm{pH} 7.5)$, and in $10 \mathrm{mM}$ sodium phosphate ( $\mathrm{pH}$ 7.5) containing $20 \mathrm{mM}$ SDS. Test compound concentrations were $100 \mu \mathrm{M}$, except for mebeverine $(50 \mu \mathrm{M})$. (a)<smiles>CC1(C)C2CCC1CC(OC(=O)C(CO)c1ccccc1)C2</smiles>

(b)<smiles>O=C(OC1CC2CCC(C1)N2F)C(CO)C1=CCCCC1</smiles>

b)<smiles>CCCN(CC)C(C)Cc1ccc(OC)cc1</smiles>

(e)<smiles>CN1CCCC1c1cccnc1</smiles>

(f)<smiles>c1ccc(Sc2ccccc2)cc1</smiles>

(g) 0

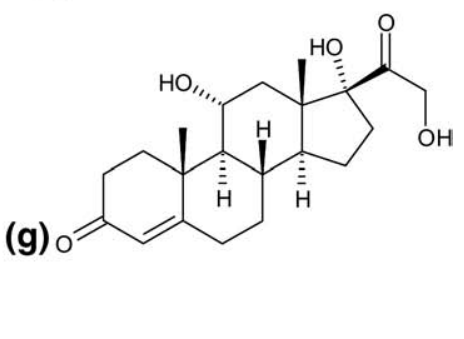

(i)

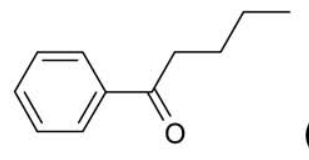

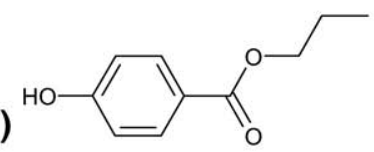

(j)<smiles>CC(C)(C)NCC(O)c1cc(O)cc(O)c1</smiles>

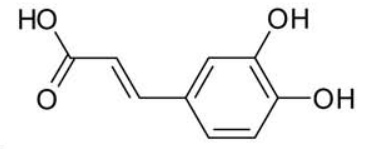

Figure 1. Molecular structures of test compounds (a) methylatropine $(\mathrm{m} / \mathrm{z} 304),(\mathbf{b})$ atropine $(\mathrm{m} / \mathrm{z} 290)$, (c) terbutaline $(\mathrm{m} / \mathrm{z} 226),(\mathbf{d})$ mebeverine $(\mathrm{m} / \mathrm{z} 430),(\mathbf{e})$ nicotine $(\mathrm{m} / \mathrm{z} 163),(\mathbf{f})$ diphenyl sulphide $(\mathrm{m} / \mathrm{z}$ 186), (g) hydrocortisone $(\mathrm{m} / \mathrm{z} 363)$, (h) valerophenone $(\mathrm{m} / \mathrm{z} 163)$, (i) propyl p-hydroxybenzoate ([M $\mathrm{H}]^{-}, m / z 179$ or $\left.[\mathrm{M}+\mathrm{H}]^{+}, m / z 181\right)$, (j) caffeic acid $\left([\mathrm{M}-\mathrm{H}]^{-}, m / z 179\right)$. 
The sodium phosphate BGE ( $\mathrm{pH}$ 7.5) was prepared by mixing $10 \mathrm{mM}$ sodium dihydrogen phosphate with 10 $\mathrm{mM}$ disodium hydrogen phosphate to the proper $\mathrm{pH}$.

For the CE-APCI-MS experiments, a test mixture of nicotine, atropine, terbutaline, hydrocortisone (each 100 $\mu \mathrm{M})$, and mebeverine $(50 \mu \mathrm{M})$ was prepared in water and analyzed using BGEs of ammonium formate $(\mathrm{pH}$ 4.0) and sodium sodium phosphate ( $\mathrm{pH}$ 3.0). The BGE of ammonium formate was prepared by adjusting 15 $\mathrm{mM}$ formic acid with $1 \mathrm{M}$ ammonium hydroxide to $\mathrm{pH}$ 4.0. The sodium phosphate BGE was prepared by mixing $10 \mathrm{mM}$ phosphoric acid with $10 \mathrm{mM}$ sodium dihydrogen phosphate to achieve $\mathrm{pH}$ 3.0. The composition of the sheath liquid was methanol-water-formic acid (75:25:0.1, vol/vol/vol), unless stated otherwise.

\section{CE System}

CE was performed using a PrinCE CE system (Prince Technologies, Emmen, The Netherlands) with a fusedsilica capillary of $50 \mu \mathrm{m}$ i.d. and a length of $90 \mathrm{~cm}$. The capillaries were flushed at 1500 mbar with $1 \mathrm{M}$ sodium hydroxide $(10 \mathrm{~min})$ and water $(10 \mathrm{~min})$ before use. During infusion experiments, the analyte solution under study was continuously introduced into the interface via the CE capillary by applying a pressure of 100 mbar to the inlet vial. No voltage was applied across the capillary during infusion to avoid differences in flow rate and analyte flux due to a generated electro-osmotic flow (EOF).

Before each CE-MS analysis, the capillary was flushed with BGE for 2 min at 1500 mbar. Injection of sample was carried out at a pressure of $35 \mathrm{mbar}$ for $6 \mathrm{~s}$, during which the nebulizing gas flow (nitrogen) of the CE-MS sprayer was switched off. A separation voltage of $30 \mathrm{kV}$ was applied for all analyses. To minimize peak broadening and loss of resolution due to the hydrodynamic flow caused by the nebulizing gas, a pressure of $\sim 70$ mbar below ambient pressure was applied at the inlet vial during CE-APCI-MS analysis.

\section{MS System}

The CE system was coupled to an Agilent Technologies 1100 Series LC/MSD SL ion-trap mass spectrometer (Waldbronn, Germany) equipped with an Agilent APCI source (Figure 2). The coupling of CE with APCI-MS was achieved through a coaxial sheath-flow CE-MS sprayer from Agilent Technologies. The sprayer was mounted on a plastic spacer with a length of $36 \mathrm{~mm}$, which was subsequently positioned on the APCI source. An electric wire grounded the sprayer to ensure a functional electrical circuit for $\mathrm{CE}$. The CE system was positioned such that the capillary inlet was at the same height as the tip of the sprayer needle to avoid siphoning effects.

During this study, the corona current was varied over the range of $0-5000 \mathrm{nA}$ and $0-10,000 \mathrm{nA}$ in the positive and negative ion mode, respectively. Using either mode, the transfer voltage (i.e., the voltage on the

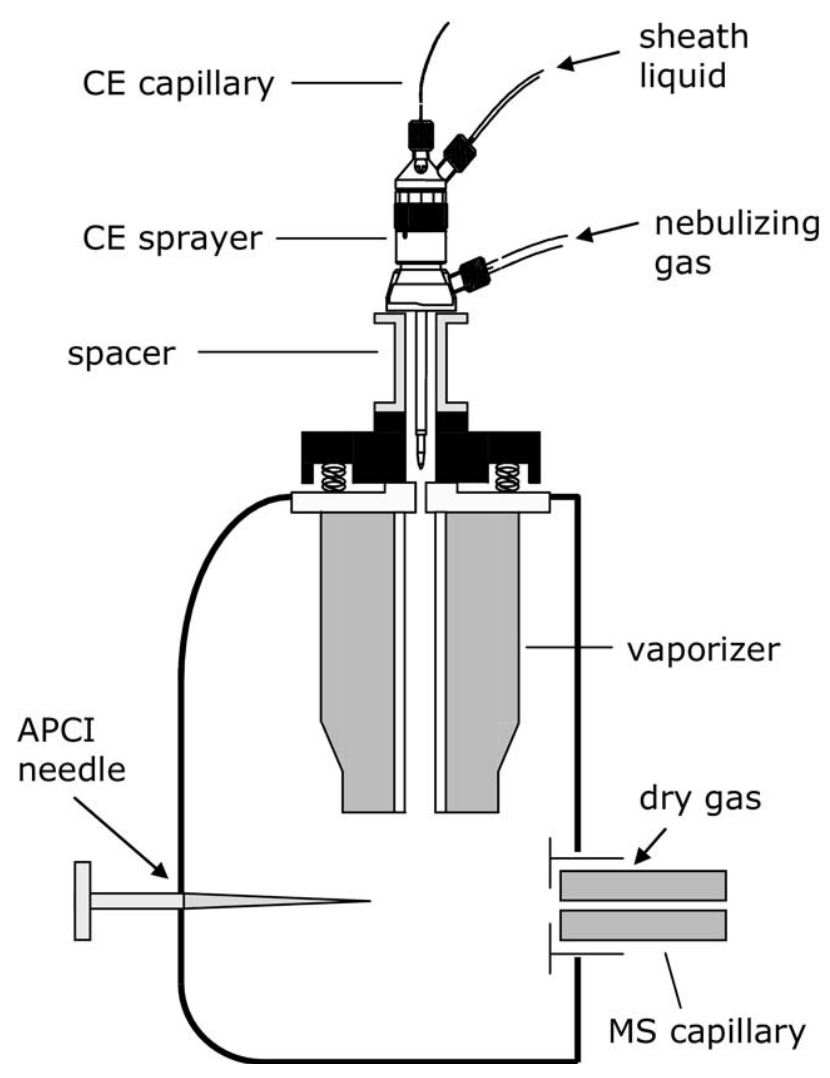

Figure 2. Schematic of the APCI source with CE sprayer.

ion sampling orifice) was studied between 0 and $5000 \mathrm{~V}$. The effect of the vaporizer temperature was examined over the range of $200-325^{\circ} \mathrm{C}$, and the sheath-liquid flow rate between 1.7 and $25 \mu \mathrm{L} / \mathrm{min}$. The drying gas temperature and flow rate were set at $300{ }^{\circ} \mathrm{C}$ and 5 $1 / \mathrm{min}$, respectively. The MS instrument was operated in positive ion mode with a scan range of $100-500 \mathrm{~m} / \mathrm{z}$, unless stated otherwise. To avoid overloading of the ion trap, the ion-charge-control option was enabled.

\section{Results and Discussion}

\section{Set-Up}

In the Agilent APCI source, the sprayer and vaporizer are aligned in such a way that the spray formation occurs orthogonally to the MS inlet. The corona-discharge needle is positioned perpendicular to the vaporizer and in-line with the MS entrance (Figure 2). The coupling of CE with APCI-MS was achieved with a sheath-flow CE-MS sprayer, which is originally designed for CE-ESI-MS. Since the APCI source is normally used in combination with a shorter, dedicated LC-APCI-MS sprayer, plastic spacers (36-41 $\mathrm{mm}$ ) were used to fit the longer CE-MS sprayer on the APCI source. To ensure a closed electrical CE circuit, an electrical wire was used to connect the $\mathrm{CE}$ sprayer with the ground of the $\mathrm{CE}$ instrument. During the initial infusion experiments, it was observed that higher analyte signal intensities were obtained when the sprayer tip was positioned as close as possible 
to the vaporizer entrance, i.e., using a short spacer. This phenomenon has also been observed in CE-APPI-MS studies with the Agilent PhotoMate APPI source [6, 7], which has a similar geometry. The reduction in analyte signal with longer spacers is probably caused by disruption of the nitrogen stream leading to a less efficient transfer of analyte into the vaporizer. Subsequent experiments were performed using the shortest spacer (36 $\mathrm{mm})$.

The test compounds for the APCI parameter study infusion experiments were selected to cover a wide range of analyte characteristics and comprised methyl atropine (quaternary ammonium), mebeverine (basic), hydrocortisone, valerophenone (neutral), diphenyl sulfide (apolar), propyl p-hydroxybenzoate (POB) (weakly acidic), and caffeic acid (acidic). Solutions of the test compounds (50-100 $\mu \mathrm{M})$ were infused through the CE capillary into the APCI source. Dry gas temperature and flow rate were set at default values for LCAPCI-MS (i.e., $300{ }^{\circ} \mathrm{C}$ and $51 / \mathrm{min}$, respectively). As a starting point, a sheath liquid consisting of methanolwater $(75: 25, \mathrm{vol} / \mathrm{vol})$ at a flow rate of $15 \mu \mathrm{L} / \mathrm{min}$ was used, and the nebulizing gas pressure and the vaporizer temperature were set 25 psi and $325^{\circ} \mathrm{C}$, respectively.

\section{Corona Current and Transfer Voltage}

To study the optimum corona discharge current, it was increased in steps of $100 \mathrm{nA}$ over the range of $0-10,000$ $\mathrm{nA}$ while infusing a test compound solution into the APCI source. During this procedure, the transfer voltage was readjusted to optimum value every increment of $1000 \mathrm{nA}$. In the positive ion mode, mebeverine, POB, valerophenone, and hydrocortisone were detected as protonated molecule. This is to be expected since the proton-affinities (PAs) of these compounds are much higher than that of the reagent gas methanol (773 $\mathrm{kJ} / \mathrm{mol}$ ) present in the sheath liquid. Hydrocortisone showed a fragment ion $(\mathrm{m} / \mathrm{z} 303)$ with a roughly similar abundance as the protonated molecule. For these compounds, a steep onset of the analyte signal was typically observed at corona currents above $1000 \mathrm{nA}$ reaching maximum intensity at about $2000 \mathrm{nA}$. Above a corona current of $2000 \mathrm{nA}$, the signal intensities decreased gradually (Figure 3a). In the positive ion mode, the transfer voltage was generally not a critical parameter and analyte signals could be achieved over the full range of $0-5000 \mathrm{~V}$. The value of the corona current at which the analyte signal onset occurred appeared to be compound-dependent. Still, these corona current values were invariably found to decrease with decreasing transfer voltage. Therefore, these two parameters have to be optimized interactively.

The apolar compound diphenyl sulfide could not be detected under any condition, neither as molecular ion nor as ammonium or sodium adduct. Apolar compounds may be ionized through charge-transfer in APCI, but as shown in LC-APCI-MS studies, abundances of molecular ions are typically very low, and


Figure 3. Signal intensity versus corona current in (a) positive ion mode and (b) negative ion mode for (a) mebeverine ([M + $\left.\mathrm{H}]^{+}, m / z 430\right)$, and (b) cafeic acid $\left([\mathrm{M}-\mathrm{H}]^{-}, m / z\right.$ 179). Sheath liquid, methanol-water $(75: 25, \mathrm{vol} / \mathrm{vol})$; transfer voltage, (a) $-4000 \mathrm{~V}$ and (b) $525 \mathrm{~V}$. Further conditions, see Experimental section.

specific conditions are needed for ionization [12, 13]. The quaternary ammonium compound methyl atropine could only be detected with a corona current of $0 \mathrm{nA}$ (i.e., performing no APCI) and a transfer voltage of 500-800 V. Under these conditions, detection of the basic compound mebeverine, which forms protonated molecules, also appeared to be possible. In a previous study on CE-APPI-MS, we observed similar phenomena when APPI was cancelled out. Ionization was attributed to a liquid-phase process similar to thermospray (TS) [14].

In the negative ion mode, the acidic compounds POB and caffeic acid were both detected as deprotonated molecules. The transfer voltage was now a critical parameter as analyte signals could only be observed at a relatively low value, slightly above the end plate offset which is fixed at $+500 \mathrm{~V}$. The influence of the corona current on signal intensity of caffeic acid is 
shown in Figure 3b. After reaching the analyte signal onset at a corona current ca. $500 \mathrm{nA}$, a more gradual increase in signal intensity was observed than in the positive ion mode. The corona current was optimal at 6000-7000 nA, after which a slight decline in signal intensity was observed with increasing current.

For a meaningful evaluation of further interface parameters (sheath liquid flow rate, nebulizing gas pressure, etc.) it is essential that the concentrations of the test compounds are within the linear response range. Using the interface parameters specified in the previous section, and mebeverine concentrations ranging from 0 to $200 \mu \mathrm{M}$, a good concentration-linearity $\left(\mathrm{r}^{2}=\right.$ $0.998, n=6$ ) was observed, indicating that the sample concentrations used in the above and further experiments $(10-100 \mu \mathrm{M})$ are suitable.

\section{Nebulizing Gas Pressure and Vaporizer Temperature}

Along with the spacer length, the nebulizing gas pressure is an important parameter for optimizing transmission of analytes into the vaporizer. In the absence of an electrical field, the nebulizer also largely controls spray formation. Signal intensities of all analytes were found to increase with nebulizing gas pressure, showing an approximate 5-fold gain when raising the pressure from 5 to 40 psi. However, increased nebulizing gas flow will also affect the CE separation. First, it brings about a siphoning effect across the CE capillary, which causes band broadening. Second, the nebulizing gas pressure influences the electrical contact between CE capillary and sprayer. High pressures may, therefore, destabilize the CE current [11]. To avoid negative effects, the nebulizing gas pressure was limited to 25 psi, which still provided a 4-fold gain in signal compared with the signal obtained at a pressure of 5 psi. It should be noted that the applied nebulizing gas pressure is significantly lower than the value of ca. 60 psi commonly used in LC-APCI-MS.

The temperature of the vaporizer was varied between 200 and $350{ }^{\circ} \mathrm{C}$. Within this range, no significant change in signal intensity was observed during continuous infusion of mebeverine $(50 \mu \mathrm{M})$. A similar result was obtained for POB, both in the positive and negative ion mode. For the less stable compound hydrocortisone, highest signal intensities were observed at $200-250{ }^{\circ} \mathrm{C}$, and fragmentation was found to increase gradually at higher temperatures. In conclusion, for CE-APCI-MS a vaporizer temperature of $250{ }^{\circ} \mathrm{C}$ was selected.

\section{Sheath-Liquid Flow Rate and Composition}

The effect of sheath liquid flow rate on signal intensity was studied in the range of $1.7-25 \mu \mathrm{L} / \mathrm{min}$ using a sheath liquid of methanol-water (75:25, vol/vol). When the flow rate was increased from 1.7 to $5 \mu \mathrm{L} / \mathrm{min}$, a steep increase in signal intensity was observed in both positive ion mode (mebeverine, $\mathrm{POB}$ ) and negative ion mode (caffeic acid, POB). Between 5 and $25 \mu \mathrm{L} / \mathrm{min}$ no significant change in signal was observed in both modes, indicating that the sheath liquid flow rate is not a critical parameter for analyte signal intensity. Nevertheless, a low sheath-liquid flow rate of $5 \mu \mathrm{L} / \mathrm{min}$ in combination with a high nebulizing gas pressure applied may result in a poor electrical contact by the sheath liquid [5], a problem that was encountered earlier by Takada et al. [15]. In the present study, the sheath liquid flow rate was set at $15 \mu \mathrm{L} / \mathrm{min}$ to provide good ionization conditions as well as a stable electrical contact.

A sheath liquid of $75 \mathrm{vol} \%$ methanol was found to give optimum results although the percentage of methanol did not seriously affect analyte signal intensities, neither in the positive ion mode, nor in the negative ion mode.

The effect of adding $0.1 \%$ formic acid or $0.125 \%$ ammonium hydroxide to the sheath liquid on analyte signals was studied both in positive and negative ion mode. Figure 4 shows the results for POB which can form positive and negative ions. In the positive ion mode, the $[\mathrm{M}+\mathrm{H}]^{+}$signal of $\mathrm{POB}$ was slightly enhanced by formic acid, whereas a strong reduction in signal was observed when ammonium hydroxide was added. As may be expected, formic acid acts as a Brønsted acid favoring analyte protonation, whereas ammonia hinders transfer of protons to analyte molecules due to its high proton-affinity (PA) $(854 \mathrm{~kJ} / \mathrm{mol})$. In the negative ion mode, formic acid strongly quenched the $[\mathrm{M}-\mathrm{H}]^{-}$signal of POB, while no POB-formate adducts were observed. Upon addition of ammonium hydroxide to the sheath liquid, a slight increase in the POB signal was observed. Again, these results can be attributed to the gas-phase acid-base properties of the above-mentioned additives. Overall, it was observed

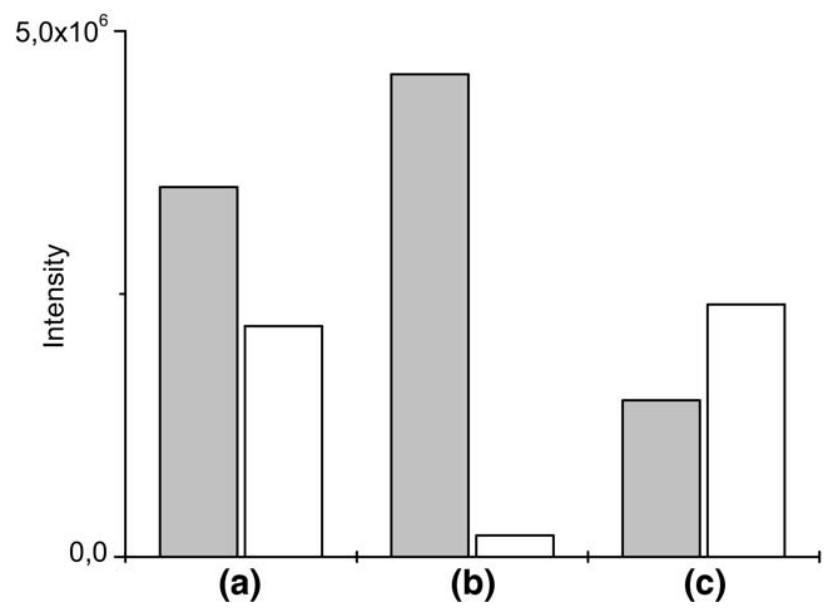

Figure 4. $\mathrm{MS}$ responses of $\mathrm{POB}$ in positive ion mode (grey bar) and negative ion mode (white bar) employing sheath liquids consisting of (a) methanol-water (75:25, vol/vol), (b) methanolwater-formic acid (75:25:0.1, vol/vol/vol), and (c) methanol-waterammonium hydroxide (75:25:0.125, vol/vol/vol). 
that analyte responses in positive and negative ion mode only modestly improved upon addition of formic acid or ammonium hydroxide, respectively. The use of these additives, however, may still be required in CE-APCI-MS, as a sufficiently conductive sheath-liquid is essential for proper CE performance.

\section{BGE Composition}

BGEs may cause interfering background signals and analyte ion suppression in MS. We studied the background spectra obtained with APCI-MS during infusion of different BGEs. In the positive ion mode, the background spectra obtained by infusion of water, $50 \mathrm{mM}$ ammonium acetate ( $\mathrm{pH}$ 6.8), $10 \mathrm{mM}$ sodium phosphate ( $\mathrm{pH}$ 7.5), and $20 \mathrm{mM}$ SDS in $10 \mathrm{mM}$ sodium phosphate ( $\mathrm{pH}$ 7.5) did not show significant peaks. This is in sharp contrast with ESI-MS, in which particularly sodium phosphate and SDS-containing BGEs have been found to give rise to abundant clusters $[8,16]$. Also in the negative ion mode, infusion of the ammonium acetate and sodium phosphate BGEs did not result in detectable background ions. When the SDS-containing BGE was employed, a strong background signal was observed for dodecyl sulphate ([SDS - Na] $]^{-}, m / z 265$ ). This ion can also be observed in the negative ion mode when ionization techniques such as ESI-MS and APPI-MS are employed [17].

The effect of the BGE composition on the signal intensities of mebeverine, valerophenone (both positive ion mode), and caffeic acid (negative ion mode) was studied. In the positive ion mode, ammonium acetate did not affect signal intensities of mebeverine and valerophenone. Ammonium adduct formation was not observed, even though the proton-affinity of valerophenone $(\sim 860 \mathrm{~kJ} / \mathrm{mol})$ is only slightly higher than that of ammonia $(854 \mathrm{~kJ} / \mathrm{mol})$. The nonvolatile BGE of sodium phosphate was also found to cause neither adduct formation nor ionization suppression. This observation is in line with findings by Takada et al. [2] who demonstrated a similar effect for caffeine, albeit using a different, laboratory-made interface and much higher analyte concentrations. In sharp contrast to these APCI results, the efficiency of ESI is known to decrease significantly in the presence of nonvolatile constituents such as sodium phosphate [2, 8]. SDS, which is often employed as a pseudo-stationary phase in micellar electrokinetic chromatography (MEKC), is an even stronger ion suppressor in ESI [18, 19]. However, the infusion experiments indicated that the addition of $20 \mathrm{mM}$ SDS did not result in reduced signal intensities in APCI-MS. For hydrocortisone, SDS even caused a slight signal increase. The enhancement of the $[\mathrm{M}+\mathrm{H}]^{+}$signal was accompanied by a reduction of the signal intensity of the fragment ion with $m / z$ 303. SDS apparently induces softer ionization conditions for this compound. In the negative ion mode, signal intensities of caffeic acid were also not affected by either sodium phosphate or SDS. For the ammonium acetate BGE a signal enhancement of $30 \%$ to $40 \%$ was observed compared with infusion of caffeic acid in water. Similar results were also obtained for POB. The presence of ammonia in the BGE presumably facilitates proton transfer reactions and by that the deprotonation of acidic compounds, which may lead to signal enhancements for this type of analytes.

\section{CE-APCI-MS}

The boundary conditions for CE-APPI-MS as follow from the studies described in the previous sections are summarized in Table 1. Specific conditions depend on the analytes to be studied.

The performance of CE-APCI-MS was evaluated using a test mixture containing four basic compounds and hydrocortisone (steroid), which was used as EOF marker. Low-pH buffers were selected to achieve a good resolution for the selected basic test compounds. Optimized interface settings were used whereas the corona current and transfer voltage were set at values that enabled proper analysis of all selected test compounds (i.e., $2700 \mathrm{nA}$ and $2800 \mathrm{~V}$, respectively). A sufficiently high conductivity of the sheath liquid was ensured by the addition of $0.1 \%$ formic acid. Figure 5 a shows typical extracted ion electropherograms (XIE) obtained with a BGE of $15 \mathrm{mM}$ ammonium formate $(\mathrm{pH}$ 4.0). The test compounds were baseline separated and typical plate numbers were in the range of 50.000140.000 , which is roughly similar to separation efficiencies previously obtained with CE-APPI-MS, but somewhat lower than achievable with CE-ESI-MS [8]. The lower separation efficiency with respect to CE-ESI-MS is largely the result of a slight hydrodynamic flow in the CE capillary due to the substantially higher nebulizing gas pressure required for APCI-MS. The detection limits for the test compounds $(\mathrm{S} / \mathrm{N}=3)$ varied between 1.6 and $10 \mu \mathrm{M}(0.7-2.0 \mu \mathrm{g} / \mathrm{mL})$ (positive ion mode), which is

Table 1. Settings of main operating APCI-MS parameters in positive and negative ion mode

\begin{tabular}{|c|c|c|}
\hline \multirow[b]{2}{*}{ Parameter } & \multicolumn{2}{|c|}{ Ion mode } \\
\hline & Positive & Negative \\
\hline Spacer length & $36 \mathrm{~mm}$ & $36 \mathrm{~mm}$ \\
\hline Corona current & $\sim 2000 \mathrm{nA}^{\mathrm{a}}$ & $\sim-6000 \mathrm{nA}$ \\
\hline Transfer voltage & $0-5000 \mathrm{~V}$ & $\sim+500 \mathrm{~V}$ \\
\hline $\begin{array}{c}\text { Nebulizing gas } \\
\text { pressure }\end{array}$ & $\geq 25 \mathrm{psi}^{\mathrm{b}}$ & $\geq 25 \mathrm{psi}^{\mathrm{b}}$ \\
\hline $\begin{array}{l}\text { Vaporizer } \\
\text { temperature }\end{array}$ & $200-350^{\circ} \mathrm{C}$ & $200-350^{\circ} \mathrm{C}$ \\
\hline Sheath liquid & & \\
\hline Flow rate & 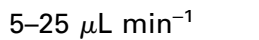 & 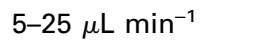 \\
\hline $\mathrm{MeOH}$ content & $0 \%-100 \%$ & $0-100 \%$ \\
\hline Additive & $0.1 \%$ Formic acid ${ }^{\mathrm{c}}$ & $0.125 \%$ Ammoniac \\
\hline BGE & $\begin{array}{l}\text { Volatile and } \\
\text { nonvolatile }\end{array}$ & $\begin{array}{l}\text { Volatile and } \\
\text { nonvolatile }\end{array}$ \\
\hline
\end{tabular}

a Settings depends on the setting of the transfer voltage.

bValue restricted by CE current stability and/or capillary siphoning effects.

${ }^{\mathrm{C}}$ May be added to increase conductivity of the sheath liquid. 


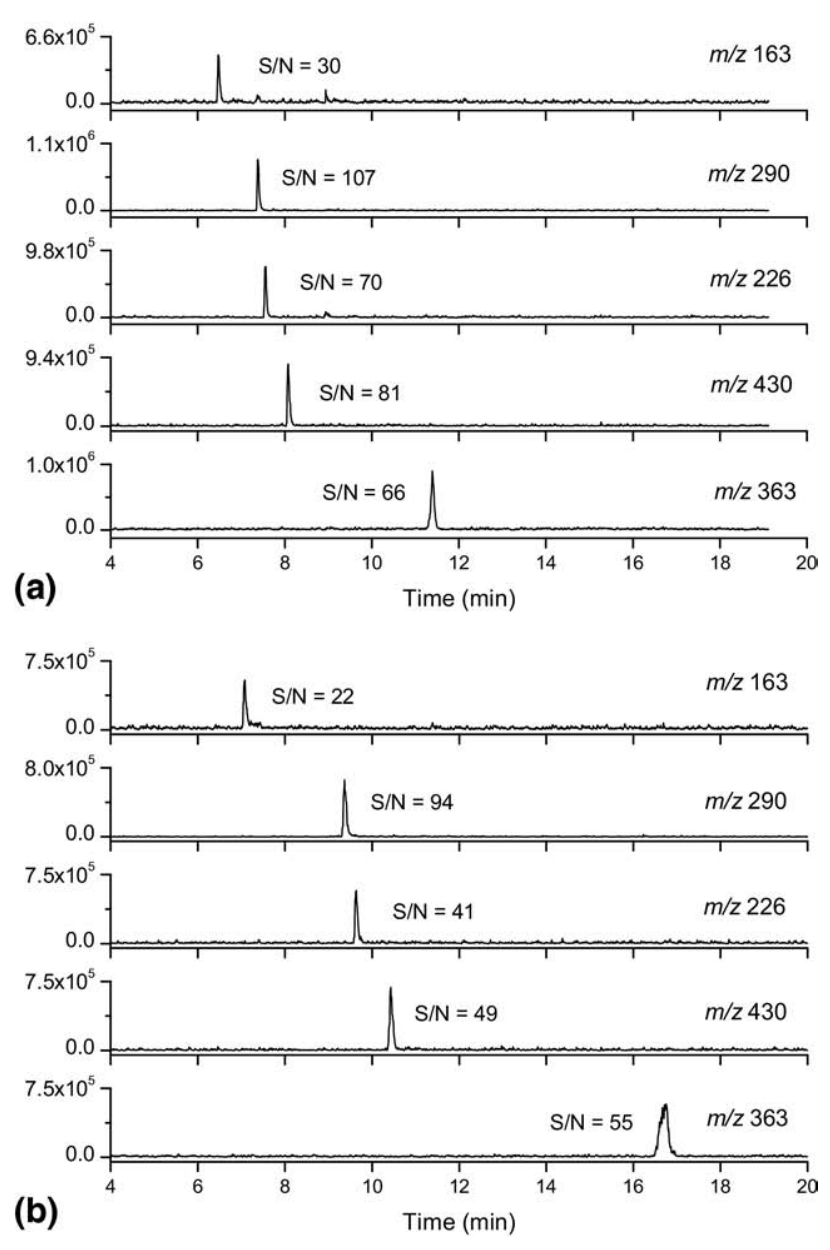

Figure 5. XIEs obtained during CE-APCI-MS of a test mixture of nicotine $(100 \mu \mathrm{M} ; m / z 163)$, atropine $(100 \mu \mathrm{M} ; \mathrm{m} / z$ 290), terbutaline $(100 \mu \mathrm{M} ; m / z$ 226), mebeverine (50 $\mu \mathrm{M} ; m / z 430)$, and hydrocortisone $(100 \mu \mathrm{M} ; \mathrm{m} / \mathrm{z}$ 363). BGE: (a) $15 \mathrm{mM}$ ammonium formate buffer ( $\mathrm{pH} 4.0$ ) and (b) $10 \mathrm{mM}$ sodium phosphate buffer ( $\mathrm{pH}$ 3.0). Interface conditions: corona current, $2700 \mathrm{nA}$; transfer voltage, $2800 \mathrm{~V}$; sheath liquid, methanol-water-formic acid (75:25:0.1, vol/ vol/vol); dry gas flow, $5 \mathrm{~L} / \mathrm{min}$; dry gas temperature, $300 \mathrm{C}$. Further conditions, see Experimental section.

more favorable than previously reported with laboratorymade APCI interfaces.

With a volatile BGE, the detection limits for basic compounds in our CE-APCI-MS set-up are similar to those obtained in CE-APPI-MS, but roughly a factor of 10 higher than with ESI-MS detection [8]. Since APCI-MS is essentially a mass-flow sensitive detector, the low flow rate in CE is relatively more suited to ESI-MS, which often behaves like a concentration-sensitive device. A further explanation for the lower sensitivity may be that the hardware of the CE-ESI-MS set-up is more specifically optimized for lower flow rates than the CE-APCI-MS set-up. For example, the dimensions of the vaporizer in the APCI source are optimized to handle flow-rates up to $2 \mathrm{~mL} / \mathrm{min}$, whereas the positioning of the CE sprayer with respect to the MS entrance in our CE-ESI-MS set-up is specifically optimized for sheath-liquid flow rates of $1-10 \mu \mathrm{L} / \mathrm{min}$.
The feasibility of using a nonvolatile BGE is demonstrated in Figure 5b, which shows CE-APCI-MS electropherograms of the above-mentioned test mixture using a BGE of $10 \mathrm{mM}$ sodium phosphate (pH 3.0). In CE-MS, the nonvolatile character of this BGE is mainly due to the substantial flux of sodium into the ion source (cathode) since the mobility of the phosphate ions is opposite to that of the EOF. The S/Ns (Figure 5) were somewhat lower than with the volatile BGE. On average there was a slight increase in noise level, although no consistent trend was observed when respective XIEs for the individual compounds were compared. Furthermore, peak heights were slightly lower than with the ammonium formate BGE. This is, at least partly, caused by increased migration times, which result in lower peak heights in CE-MS [8]. Taking this into account, it can be concluded that no, or hardly any, ionization suppression occurs when the nonvolatile BGE is employed. This shows that APCI is clearly more resistant towards nonvolatile BGE constituents than ESI $[2,8,19]$.

\section{Conclusions}

The present paper has investigated the possibility of CE-APCI-MS using an orthogonal interface set-up. This coupling required specific optimization, especially because $\mathrm{CE}$ entails low flow rates and the requirement of a closed electrical circuit. In the positive ion mode, the optimum setting of the corona current was found to depend on the transfer voltage, which means that in our set-up these parameters have to be optimized simultaneously for optimal analyte response. Other parameters, such as sheath-liquid composition and flow rate, and vaporizer temperature only have a modest effect on analyte signals. These parameters can therefore be optimized with respect to the separation performance and stability of the CE current. Another favorable characteristic of APCI-MS is its strong resistance towards ionization suppression by nonvolatile BGEs and SDS, thereby showing good potential for the coupling of MEKC with MS.

The detection limits achieved with the presented CE-APCI-MS system are within the high $\mathrm{ng} / \mathrm{mL}$ to low $\mu \mathrm{g} / \mathrm{mL}$ range, which is more favorable than previously reported systems. The improved performance is likely to be due to higher sample transmission through the vaporizer, and/or a lower noise level resulting from the orthogonal set-up. A further improvement in sensitivity may be achieved by adapting the APCI source to the low flow rates encountered in CE-MS. Indeed, the strong effects of the spacer length and nebulizing gas pressure on signal responses seem to indicate that sample transmission through the vaporizer is the most critical step in our CE-APCI-MS set-up. It may therefore be advantageous to achieve the CE-MS coupling through a Multimode ESI/APCI source, which lacks a long vaporizer tube, and instead uses an infrared emitter for sample vaporization. As an additional advantage, the multimode source requires a lower nebulizing gas pressure ( $20 \mathrm{psi})$ in 
LC-MS than the dedicated APCI source (60 psi), which would reduce siphoning effects and thus enhance separation efficiency in CE-MS.

\section{Acknowledgments}

The authors acknowledge financial support of this work by AstraZeneca, Global Process R and D, External Science Group (Macclesfield, UK).

\section{References}

1. Olivares, J. A.; Nguyen, N. T.; Yonker, C. R.; Smith, R. D. On-Line Mass Spectrometric Detection for Capillary Zone Electrophoresis [1]. Anal. Chem. 1987, 59, 1230-1232.

2. Takada, Y.; Sakairi, M.; Koizumi, H. Atmospheric Pressure Chemical Ionization Interface for Capillary Electrophoresis/Mass Spectrometry. Anal. Chem. 1995, 67, 1474-1476.

3. Muijselaar, P. G.; Otsuka, K.; Terabe, S. On-Line Coupling of PartialFilling Micellar Electrokinetic Chromatography with Mass Spectrometry. I. Chromatogr. A 1998, 802, 3-15.

4. Isoo, K.; Otsuka, K.; Terabe, S. Application of Sweeping to Micellar Electrokinetic Chromatography-Atmospheric Pressure Chemical IonizationMass Spectrometric Analysis of Environmental Pollutants. Electrophoresis 2001, 22, 3426-3432.

5. Tanaka, Y.; Otsuka, K.; Terabe, S. Evaluation of an Atmospheric Pressure Chemical Ionization Interface for Capillary ElectrophoresisMass Spectrometry. J. Pharm. Biomed. Anal. 2003, 30, 1889-1895.

6. Nilsson, S. L.; Andersson, C.; Sjöberg, P. J. R.; Bylund, D.; Petersson, P.; Jörntén-Karlsson, M.; Markides, K. E. Phosphate Buffers in Capillary Electrophoresis-Mass Spectrometry Using Atmospheric Pressure Photoionization and Electrospray Ionization. Rapid Commun. Mass Spectrom. 2003, 17, 2267-2272.

7. Mol, R.; De Jong, G. J.; Somsen, G. W. On-Line Capillary ElectrophoresisMass Spectrometry Using Dopant-Assisted Atmospheric Pressure Photoionization: Setup and System Performance. Electrophoresis 2005, 26, $146-154$.
8. Hommerson, P.; Khan, A. M.; De Jong, G. J.; Somsen, G. W. Comparison of Atmospheric Pressure Photoionization and ESI for CZE-MS of Drugs. Electrophoresis 2007, 28, 1444-1453.

9. Schappler, J.; Guillarme, D.; Prat, J.; Veuthey, J.-L.; Rudaz, S. Coupling CE with Atmospheric Pressure Photoionization MS for Pharmaceutical Basic Compounds: Optimization of Operating Parameters. Electrophoresis 2007, 28, 3078-3087.

10. Mol, R.; De Jong, G. J.; Somsen, G. W. Atmospheric Pressure Photoionization for Enhanced Compatibility in On-Line Micellar Electrokinetic Chromatography-Mass Spectrometry. Anal. Chem. 2005, 77, 5277-5282.

11. Himmelsbach, M.; Haunschmidt, M.; Buchberger, W.; Klampfl, C. Microemulsion Electrokinetic Chromatography with On-Line Atmospheric Pressure Photoionization Mass Spectrometric Detection. Anal. Chem. 2007, 79, 1564-1568.

12. Kolakowski, B. M.; Grossert, J. S.; Ramaley, L. The Importance of Both Charge Exchange and Proton Transfer in the Analysis of Polycyclic Aromatic Compounds Using Atmospheric Pressure Chemical Ionization Mass Spectrometry. J. Am. Soc. Mass Spectrom. 2004, 15, 301-310.

13. Perazzolli, C.; Mancini, I.; Guella, G. Benzene-Assisted AtmosphericPressure Chemical Ionization: A New Liquid Chromatography-Mass Spectrometry Approach to the Analysis of Selected Hydrophobic Compounds. Rapid Commun. Mass Spectrom. 2005, 19, 461-469.

14. Hommerson, P.; Khan, A. M.; Bristow, T.; Niessen, W.; De Jong, G. J. Somsen, G. W. Photon-Independent Gas-Phase Ion Formation in Capillary Electrophoresis-Mass Spectrometry Using Atmospheric Pressure Photoionization. Anal. Chem. 2007, 79, 5351-5357.

15. Takeda, S.; Tanaka, Y. Yamane, M.; Siroma, Z.; Wakida, S.; Otsuka, K. Terabe, S. Ionization of Dichlorophenols for Their Analysis by Capillary Electrophoresis-Mass Spectrometry. J. Chromatogr. A 2001, 924, 415-420.

16. Hommerson, P.; Khan, A. M.; De Jong, G. J.; Somsen, G. W. Comparison of Electrospray Ionization and Atmospheric Pressure Photoionization for Coupling of Micellar Electrokinetic Chromatography with Ion Trap Mass Spectrometry. J. Chromatogr. A 2008, 1204, 197-203.

17. Schappler, J.; Guillarme, D.; Rudaz, S.; Veuthey, J.-L. Microemulsion Electrokinetic Chromatography Hyphenated to Atmospheric Pressure Photoionization Mass Spectrometry. Electrophoresis 2008, 29, 11-19.

18. Rundlett, K. L.; Armstrong, D. W. Mechanism of Signal Suppression by Anionic Surfactants in Capillary Electrophoresis-Electrospray Ionization Mass Spectrometry. Anal. Chem. 1996, 68, 3493-3497.

19. Somsen, G. W.; Mol, R.; De Jong, G. J. On-line Micellar Electrokinetic Chromatography-Mass Spectrometry: Feasibility of Direct Introduction of Nonvolatile Buffer and Surfactant into the Electrospray Interface. J. Chromatogr. A 2003, 1000, 953-961. 